\title{
Successful surgical excision of cerebral abscess caused by Fonsecaea monophora in an immunocompetent patient
}

Radim Dobias ${ }^{1,2}$, Michal Filip ${ }^{3,4}$, Katerina Brajzova ${ }^{5}$, Dagmar Dolinska ${ }^{6}$, Ales Dujka ${ }^{7}$, Petr Linzer ${ }^{3}$, Patrik Jurek ${ }^{3}$, Barbora

Studena ${ }^{6}$, Eva Cerna ${ }^{5}$, Jakub Mrazek ${ }^{8}$, Pavla Jaworska ${ }^{1}$, Michaela Kantorova ${ }^{8}$, Vit Hubka ${ }^{9,10}$

${ }^{1}$ Department of Bacteriology and Mycology, Institute of Public Health in Ostrava, Ostrava, Czech Republic; ${ }^{2}$ Department of Microbiology, Faculty of Medicine and Dentistry, Palacky University Olomouc, Olomouc, Czech Republic; ${ }^{3}$ Neurosurgery, Tomas Bata Regional Hospital, Zlin, Czech Republic; ${ }^{4}$ Department of Rehabilitation, Faculty of Medicine, University of Ostrava, Ostrava, Czech Republic; ${ }^{5}$ Department of Infectious Diseases, Uherske Hradiste Hospital, Uherske Hradiste, Czech Republic; ${ }^{6}$ Pathological and Anatomical Department, Tomas Bata Regional Hospital, Zlin, Czech Republic; ${ }^{7}$ Radiodiagnostic Department, Uherske Hradiste Hospital, Uherske Hradiste, Czech Republic; ${ }^{8}$ Department of Molecular Biology, Institute of Public Health in Ostrava, Ostrava, Czech Republic; ${ }^{9}$ Department of Botany, Faculty of Science, Charles University, Prague, Czech Republic; ${ }^{10}$ Laboratory of Fungal Genetics and Metabolism, Institute of Microbiology of the AS CR, Prague, Czech Republic

Cerebral abscesses caused by dark-pigmented Fonsecaea fungi are rare, especially in otherwise completely healthy individuals. A 61-year-old man from Moldova, living in the Czech Republic, who had worked as a locksmith on oil platforms in Turkmenistan, Kazakhstan, Sudan and Iraq since 1999, was admitted to a neurology ward for a sudden disorder angularity of the right leg, dysarthria and hypomimia on 10th August 2017. In July that year, the patient began to confuse names but was afebrile, had no infection and was generally well. Six days before the admission, he was diagnosed with erosive gastritis and started on outpatient treatment with proton pump inhibitors (PPIs). Previously, he had only been treated for essential hypertension, he had never had surgery or a serious injury, and he had worked for 12 hours a day. During his stay in Sudan, he had undergone bilateral myringotomy to treat his ear pain and hearing problems.

During his hospitalization, central paresis of right cranial nerves VII and XII, severe right hemiparesis to hemiplegia were diagnosed by neurological examination. A brain CT scan revealed a cystic mass with an irregular wall and marked peripheral edema. Antiedema therapy and PPI were administered. Magnetic resonance imaging showed frontally, temporally and parietally significant peripheral edema on the background of the bearing of size $3 \times 1$ and $5 \times 2 \mathrm{~cm}$ (see Fig. $1 \mathrm{a}-\mathrm{d}$ ). Inflammatory biomarkers and blood count were normal, and HIV tests were negative.

On 15th August 2017, the intracranial bearing was excised under intraoperative SonoWand guidance. Tissue samples were histologically positive for dark-pigmented hyphae, suggesting dematiaceous fungi (see Fig. 1 e-k). Therefore, amphotericin therapy was initiated immediately. By direct detection and identification of DNA from brain tissue by PCR, the species $F$. monophora was provisionally identified. The ITS1-5.8S-ITS2 rDNA region was used for species identification. After 6-days culturing of brain tissue, the previously identified F. monophora was confirmed by macro- and micromorphological analyses and by sequencing of the ITS15.8S-ITS2 rDNA region from the cultured mycelium. The strain isolated from brain tissue was found to be in vitro sensitive to voriconazole ( $\mathrm{MIC}=0.016 \mathrm{mg} / \mathrm{L}$ ) and resistant to amphotericin ( $\mathrm{MIC}=4,000 \mathrm{mg} / \mathrm{L}$ ) by the E-test method. Therefore, amphotericin was replaced with voriconazole. Prior to and during antifungal therapy, (1-3)- $\beta$-D-glucan (BG) concentrations in the patient's serum were measured to monitor possible invasion of residual fungal particles into the surrounding tissue after the surgery. Initially, BG concentration rose to positive values $(149 \mathrm{pg} / \mathrm{mL})$; during therapy, concentration of this panfungal biomarker fell to negative values $(16 \mathrm{pg} / \mathrm{mL})$. The postoperative finding did not require additional surgery.
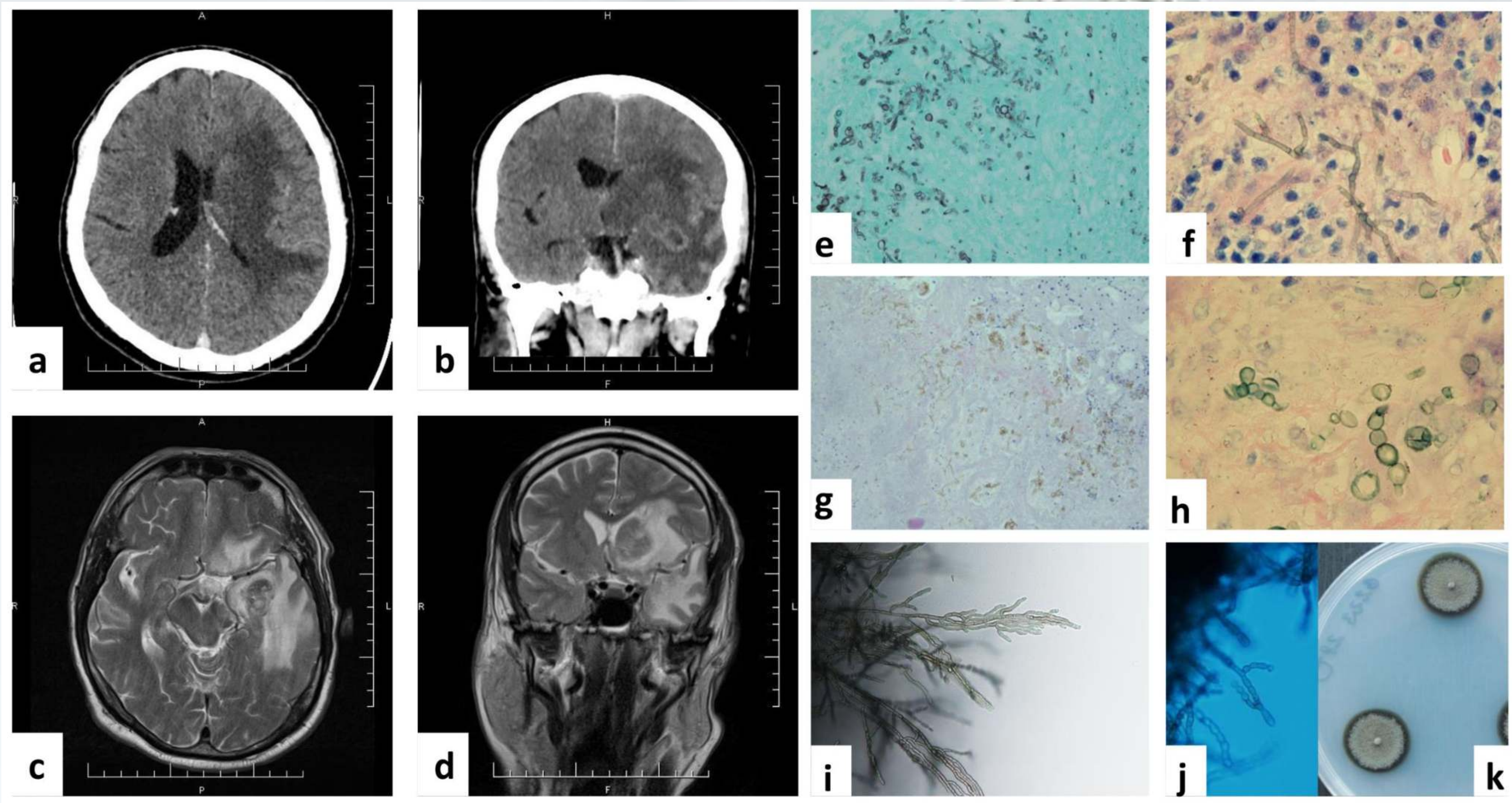

Fig. 1 Transverse CT scan view of peripheral edema affecting the right side of the left lateral brain compartment (a), Frontal CT scan view of the brain abscess (b) Transverse magnetic resonance (MR) imaging view of the left expansion (size $3 \times 1$ and $5 \times 2 \mathrm{~cm}$ ) (c), Frontal MR imaging view of the abscess position (d), Microscopic image of fungal particles shown by Grocott's methenamine silver staining (e), Microscopic image of dark pigmented hyphae shown by Giemsa-Romanowski staining (f), Microscopic image of fungal particles shown by hematoxylin and eosin staining (g), Detail of microscopic fungal particles shown by Giemsa-Romanowski staining ( $\mathbf{h}$ ), Fonsecaea monophora - Branched hyphae in microculture on Sabouraud agar (i), F. monophora - one-celled, ellipsoidal to fusiform, dry conidia arising through blastic, acropetal conidiogenesis and arranged in branched chains shown by lactophenol cotton blue staining (j), F. monophora - 7-day-old culture on Czapek yeast agar incubated at $28^{\circ} \mathrm{C}(\mathbf{k})$ 